\title{
Critical IIlness Scoring Systems: Sequential Organ Failure Assessment, Acute Physiology and Chronic Health Evaluation II, and Quick Sequential Organ Failure Assessment to Predict the Clinical Outcomes in Scrub Typhus Patients with Organ Dysfunctions
}

\author{
Prasanth Balasubramanian, Navneet Sharma, Manisha Biswal' ${ }^{1}$ Ashish Bhalla, Susheel Kumar, Vivek Kumar ${ }^{2}$ \\ Departments of Internal Medicine, ${ }^{1}$ Microbiology and ${ }^{2}$ Nephrology, PGIMER, Chandigarh, India
}

\section{Abstract}

Background and Aim: Scrub typhus (ST) is an acute infectious disease of variable severity caused by Orientia (formerly Rickettsia) tsutsugamushi. The disease can be complicated by organ dysfunctions and the case fatality rate (CFR) is approximately $15 \%$, which further rises with the development of severe complications. We studied the clinical features of the ST and the performance of critical illness scoring systems (CISSs) - Acute Physiology and Chronic Health Evaluation (APACHE) II, sequential organ failure assessment (SOFA), and quick SOFA (qSOFA) in predicting the clinical outcomes in complicated ST (cST) patients admitted to the emergency department. Study Design and Methods: A prospective observational study was done in 50 patients diagnosed to have cST with one or more organ dysfunctions. Clinical features and laboratory parameters were recorded and the patients were followed up until the end of their stay in the hospital. APACHE II, SOFA, and qSOFA scores at admission were calculated and were analyzed in predicting the clinical outcomes. Results: The median SOFA, APACHE II, and qSOFA scores of the cohort were 7 (interquartile range [IQR] $=13-22$ ), 8 (IQR $=5-11$ ), and $2(\mathrm{IQR}=1-3)$, respectively. The median duration of in-hospital stay was 9 (IQR 5-11) days and overall CFR was $8 \%$. On bivariate analysis, both SOFA $(P=0.031)$ and qSOFA $(P=0.001)$ predicted mortality. However, only SOFA score correlated with the in-hospital stay duration (Pearson's correlation $=0.311, P=0.028$ ). Conclusion: Among the three CISSs studied, the SOFA score correlated with in-hospital stay duration and mortality, whereas the qSOFA score formed a simple as well as a convenient tool in predicting the mortality in patients of cST with organ dysfunction.

Keywords: Acute Physiology and Chronic Health Evaluation II, organ dysfunction, quick sequential organ failure assessment, scrub typhus, sequential organ failure assessment

\section{INTRODUCTION}

Scrub typhus (ST) is a mite-borne acute infectious disease of variable severity caused by Orientia (formerly Rickettsia) tsutsugamushi and is primarily distributed in the Asia-Pacific rim. Every year, around a million cases are reported and around a billion people are at the risk of the infection. There is a reported increase in the prevalence of the disease in the recent years following urbanization of the rural areas.

ST is transmitted to humans by the bite of "chiggers," which are the larval stage of the trombiculid mites. The incubation

\begin{tabular}{|l|l|}
\hline \multicolumn{3}{|c|}{ Access this article online } \\
\hline Quick Response Code: & Website: \\
\hline & www.ijccm.org \\
\hline & \\
\hline
\end{tabular}

period is usually around 7-21 days. An eschar may be found at the site of the chigger bite. The disease may start with constitutional symptoms such as headache and myalgia or

Address for correspondence: Dr. Navneet Sharma, Department of Internal Medicine, Room No-16, IV Floor, F Block, Nehru Hospital, PGIMER, Sector 12, Chandigarh - 160 012, India. E-mail: navneetsharma@hotmail.com

This is an open access journal, and articles are distributed under the terms of the Creative Commons Attribution-NonCommercial-ShareAlike 4.0 License, which allows others to remix, tweak, and build upon the work non-commercially, as long as appropriate credit is given and the new creations are licensed under the identical terms.

For reprints contact: reprints@medknow.com

How to cite this article: Balasubramanian P, Sharma N, Biswal M, Bhalla A, Kumar S, Kumar V. Critical illness scoring systems: Sequential organ failure assessment, Acute Physiology and Chronic Health Evaluation II, and quick sequential organ failure assessment to predict the clinical outcomes in scrub typhus patients with organ dysfunctions. Indian J Crit Care Med 2018;22:706-10. 
with sudden-onset fever and can be complicated by organ dysfunctions, including acute kidney injury (AKI), acute liver failure, acute meningoencephalitis, thrombocytopenia, and acute respiratory distress syndrome (ARDS). The reported case fatality rate (CFR) is approximately $15 \%$ and rises with the development of severe complications. ${ }^{[1]}$

The severity and outcome of many infectious and inflammatory diseases are usually predicted with the use of critical illness scoring systems (CISSs). Some of these are the Acute Physiology and Chronic Health Evaluation (APACHE II), sequential organ failure assessment (SOFA), and quick SOFA (qSOFA) and they are used in intensive care units (ICUs) worldwide. A higher score heralds a poor outcome and the necessity of treatment in an intensive care facility.

The SOFA scoring, due to the ease of use, is commonly used in predicting the need for admission to ICU and outcome of the disease. ${ }^{[2]}$ The APACHE II scoring system was first introduced by Knaus et al. and serves as a useful index for the evaluation of the severity of the disease due to the associated multiple organ systems involvement. ${ }^{[3]}$ The qSOFA score was introduced by Seymour et al. in 2016. It includes only three parameters; the presence or absence of respiratory rate $\geq 22 / \mathrm{min}$, systolic blood pressure $<100 \mathrm{mmHg}$, and altered mentation. It can be calculated bedside for the patients outside the ICU and can correlate with the risk of dying of sepsis. ${ }^{[4]}$

A review of the available medical literature yielded only a single study that has used CISSs to predict the outcome of ST ${ }^{[5]}$ In this study, we compared three CISSs; the APACHE II, SOFA, and qSOFA in predicting the outcome of this infectious disease.

\section{Methods}

A prospective observational study was conducted over a period of 12 months from June 2015 to May 2016 in the Department of Medical Emergency. The inclusion criteria were an acute febrile illness (temperature $>38.2^{\circ} \mathrm{C}$ on multiple occasions) with IgM antibodies against $O$. tsutsugamushi by ELISA (InBios International, Inc., Seattle, WA, USA) or/and nested polymerase chain reaction positivity in blood or from eschar tissue with any organ dysfunctions. ${ }^{[6]}$

Of 212 screened patients, 50 patients of complicated ST (cST) were enrolled in the study and admitted to the emergency medical ward. A detailed history and a general physical examination were carried out at all. A special emphasis was made to document the presence of eschar and rash. Hematological investigations carried out were hemoglobin, complete blood count along with differential leukocyte count, and peripheral blood film. The serum biochemical tests done were serum sodium and potassium, renal function tests which include blood urea and serum creatinine, and liver function tests which include serum bilirubin, serum glutamic oxaloacetic transaminase (SGOT), and serum glutamic pyruvic transaminase (SGPT). Others serum biochemical tests including serum amylase, lipase, total protein and albumin, calcium, and phosphorus were done wherever indicated. An arterial blood gas analysis was done to determine $\mathrm{pH}, \mathrm{PaO}_{2}, \mathrm{HCO}_{3}$ and $\mathrm{PaCO}_{2}$. A rapid diagnostic card test (Nicholas Piramal kit, SD Bioline, India) for malaria and dengue (NS1 and IgM using Panbio Dengue Duo Cassette by Alere Medical, USA) were carried out. The Widal test and two sets of blood cultures were sent and IgM ELISA to detect antibodies against Leptospira was carried out. Those cases presenting as acute encephalitis syndrome underwent neuroimaging (computed tomography [CT] scan head and/or magnetic resonance imaging brain) followed by cerebrospinal fluid analysis. Radiological investigations carried out were chest X-ray, ultrasonography abdomen, and/or contrast-enhanced CT chest and/or abdomen whenever indicated. All patients underwent scoring with the APACHE II, SOFA, and qSOFA scores.

After admission to the emergency medical ward, all patients were managed with standard care as per the guidelines of the "Surviving Sepsis Campaign."[7] ARDS was defined by "Berlins" definition, AKI by KDIGO guidelines, thrombocytopenia at platelet (Plt) counts $<1.5 \times 10^{5} / \mu \mathrm{L}$ and severe thrombocytopenia by counts $<20 \times 10^{5} / \mu \mathrm{L}$, acute encephalitis by the presence of fever and a change in mentation, and/or seizures and acute liver dysfunction by a greater than 2-fold rise of serum transaminases with or without hyperbilirubinemia. ${ }^{[8-12]}$ The need of supportive therapy, which includes the need for ventilator support, dialysis, inotropes, intravenous fluids, and blood components, was recorded on the case record forms. The duration of hospital stay and the outcome were also duly noted.

\section{Ethics statement}

The study was carried out with prior approval of the institutional ethics committee board. Written informed consent was obtained from all the adult subjects who were capable of giving consent or from the next of kin where the patient was incapable of giving the informed consent due to severity of the illness. For all the subjects with age group between 12 and 17 years, informed consent was obtained from any of their parents or legal guardian.

\section{Statistical analysis}

The normality of quantitative variables was checked applying Kolmogorov-Smirnov test. For normally distributed data, mean \pm standard deviation was calculated for all continuous data; otherwise, median (interquartile range [IQR]), frequency, percentage, and proportions were calculated for qualitative variables. Mann-Whitney U-test was used to compare mean difference in nonparametric data, and Student's $t$-test was used to compare mean difference in parametric data. Different cutoff values for SOFA, APACHE II, and qSOFA scores were used for predicting mortality. Of all cutoff values, the best cutoff value was selected calculating sensitivity (Se), specificity (Sp) obtained from the receiver operator characteristic (ROC) curve. A $P<0.05$ was considered to be of statistically significance. The relationship between SOFA, APACHE II, 
and qSOFA scoring system with duration of hospital stay and other outcomes were measured with Spearman correlation coefficient. SPSS Statistical Package for the Social Sciences version 22 (IBM corporation, Chicago, IL, USA) was used for the data analysis.

\section{RESULTS}

Fifty patients with ST were eligible for the study with male-to-female ratio of 1.5:1. All patients presented in the post-monsoon season from July to October. The baseline characteristics of the study group have been mentioned in Table 1.

On analyzing the laboratory parameters at admission, anemia $(\mathrm{Hb}<13 \mathrm{mg} / \mathrm{dL})$ was present in 42 patients $(84 \%)$, leukocytosis (total leukocyte count [TLC] $>11,000$ ) in 18 patients $(36 \%)$, leukopenia (TLC $<4000)$ in four patients (8\%), thrombocytopenia (Plt count $<150 \times 10^{3} / \mathrm{cu} \mathrm{mm}$ ) in 41 patients $(82 \%)$ with severe thrombocytopenia (Plt count $<50 \times 10^{3} / \mathrm{cu} \mathrm{mm}$ ) in $38 \%$, hyperuricemia (blood urea nitrogen $>20 \mathrm{mg} / \mathrm{dL})$ in 34 patients $(68 \%)$, serum creatinine $>1.2 \mathrm{mg} / \mathrm{dL}$ in $48 \%$ patients, hyperbilirubinemia (serum bilirubin $>1 \mathrm{mg} / \mathrm{dL}$ ) in 29 patients (58\%), and SGOT and SGPT greater than 3 times upper limit of normal in 24 patients $(48 \%)$. Fifteen patients $(30 \%)$ received intravenous doxycycline and 35 patients $(70 \%)$ received parenteral azithromycin.

\section{Complications and outcomes}

The frequency of various complications and the median duration of hospital stay have been mentioned in Table 2. Blood products used in five patients $(10 \%)$ were as follows: packed red blood cells in two patients and random donor Plts for severe thrombocytopenia in three patients. Fifteen patients $(30 \%)$ required vasopressor support for shock out of which, in nine patients, double inotropic support (noradrenaline and dobutamine) was administered. Thirty-nine cases developed ARDS, of which 22 required ventilatory assistance with a non-rebreathing mask and 17 required invasive mechanical ventilation (mean 6 days, IQR 5-10 days). There were 21 cases (42\%) of AKI, of which eight cases (16\%) underwent hemodialysis. Four patients died with a CFR of $8 \%$ [Table 2].

The bivariate analysis of various characteristics between survivors and nonsurvivors has been shown in Table 3. Of all the three CISSs, only the SOFA scores showed a linear correlation with the in-hospital stay duration (Pearson's correlation $=0.311, P=0.028)$. On analyzing ROC curves, the area under the curves (AUC) for SOFA and APACHE II was 0.764 and 0.747 with a Se of $75 \%$ and $75 \%$ and Sp of $67 \%$ and $76 \%$, respectively, for a cutoff of 9.5 and 21.5. qSOFA showed Se of $100 \%$ and $0 \%$ with $\mathrm{Sp}$ of $41 \%$ and $91 \%$ when the cutoff scores were 1.5 and 2.5 , respectively, with AUC of 0.663 .

\section{Discussion}

ST is an important cause of acute febrile illness in Southeast Asia-Pacific region. The pathophysiology of the disease is

\begin{tabular}{lc}
\hline \multicolumn{2}{l}{ Table 1: Baseline characteristics of the study group } \\
( $\boldsymbol{n}=\mathbf{5 0 )}$ & Value \\
\hline Variable & $34.2(12-70)$ \\
\hline Age, median (IQR), years & $1.27: 1$ \\
Male:female & $8(5-11)$ \\
Duration of stay in days, median (IQR) & \\
Symptoms (\%) & $45(90)$ \\
Fever & $32(64)$ \\
Dyspnea & $16(32)$ \\
Altered sensorium & $14(28)$ \\
Jaundice & $13(26)$ \\
Abdominal pain & $10(20)$ \\
Vomiting & $8(16)$ \\
Decreased urine output & $3(6)$ \\
Eschar & $2(4)$ \\
Rash & $2(4)$ \\
Nausea & \\
Physical findings (\%) & $10(20)$ \\
Pallor & $12(24$ \\
Hypotension & $4(8)$ \\
Lymphadenopathy & $20(40)$ \\
Crackles & $23(46)$ \\
Hepatomegaly & $12(24)$ \\
Splenomegaly & $8(5-11)$ \\
Admission median SOFA score (IQR) & $17(13-22)$ \\
Admission median APACHE-II score (IQR) & $2(1-3)$ \\
Admission median qSOFA score (IQR) & \\
\hline IQR: Interquartile range; SOFA: Sequential organ failure assessment; \\
qSOFA: Quick SOFA; APACHE-II: Acute Physiology and Chronic \\
Health Evaluation-II
\end{tabular}

Table 2: Complications and outcomes of the study group $(n=50)$

\begin{tabular}{lc}
\hline Parameter & Frequency (\%) \\
\hline Thrombocytopenia & $41(82)$ \\
ARDS & $33(66)$ \\
AKI & $21(42)$ \\
Acute encephalitis syndrome & $9(18)$ \\
ALF & $3(6)$ \\
Discharge & $46(92)$ \\
Death & $4(8)$ \\
Duration of hospital stay (IQR) & 8 days $(5-11)$ \\
\hline
\end{tabular}

ARDS: Acute respiratory distress syndrome; AKI: Acute kidney injury; ALF: Acute liver failure; IQR: Interquartile range

mainly a vasculitis, and the resulting multi-organ dysfunction manifests as disseminated intravascular coagulation, shock, pulmonary edema, meningoencephalitis, and hepatic dysfunction. ${ }^{[13]}$

The male-to-female ratio was $1.4: 1$ in our study which is similar to other studies from South India. ${ }^{[14-19]}$ A similar male preponderance has also been seen in other studies from the Asia-Pacific region outside India except South Korea (male-to-female ratio of 1:1.6), a trend that may be influenced by the prevailing socioeconomic culture of females occupied with farming. ${ }^{[20-24]}$ 


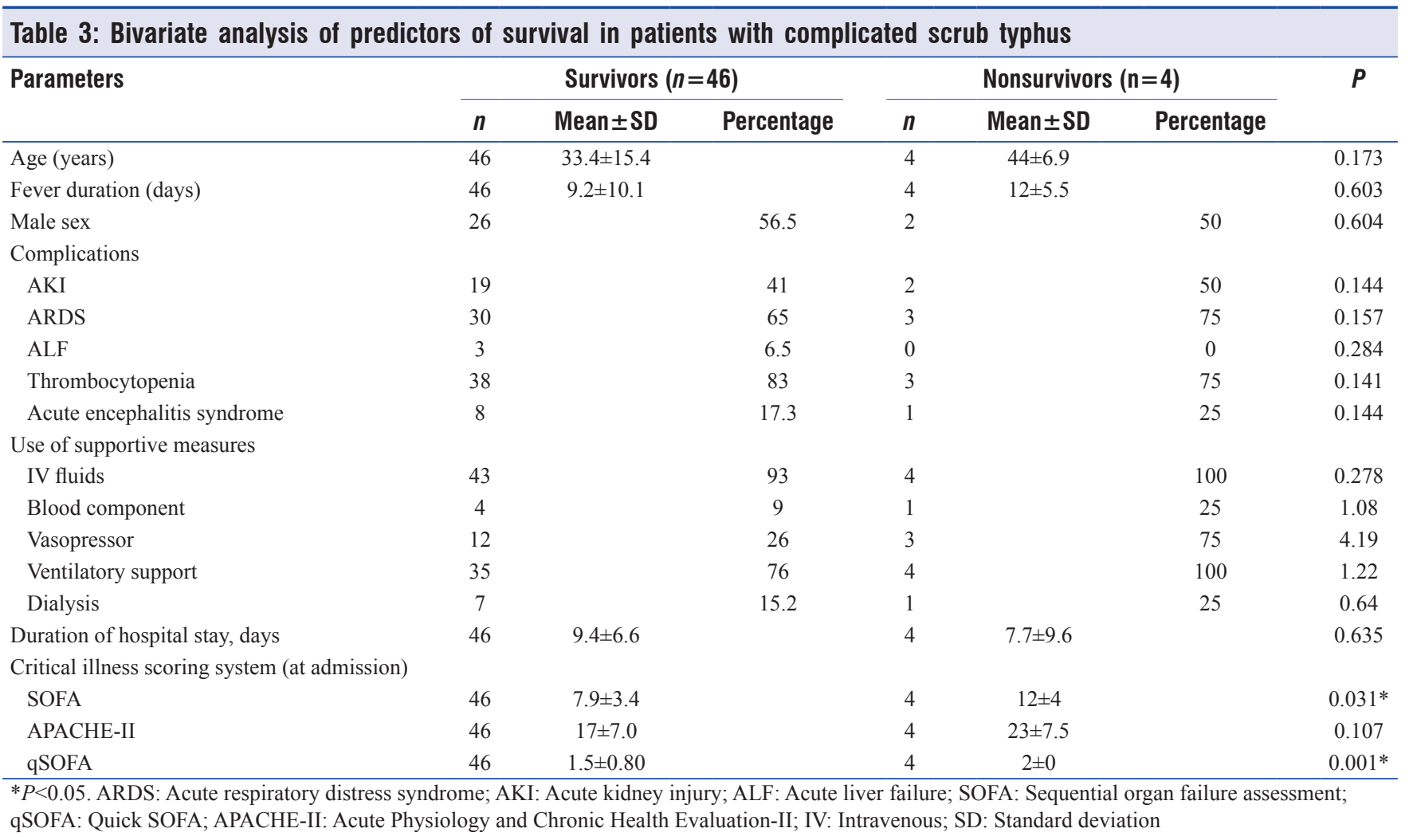

A majority of our cases presented from July to October, a period corresponding to the postmonsoon season after maximum rainfall. Most of the cases were from the surrounding state of Himachal Pradesh (34\%) in the Sub-Himalayan belt and the remaining from Haryana (28\%), Punjab (12\%), Chandigarh (12\%), Uttar Pradesh (6\%), Jammu and Kashmir (2\%), Uttarakhand (2\%), Bihar (2\%), and Jharkhand (2\%). This distribution of cases emphasizes the fact that the disease is no longer confined to forested areas and has become urban and semi-urban. The causative factor attributable to this paradigm shift of ST is a change in the ecosystem brought about by the destruction of forested areas. ${ }^{[14]}$

Of the clinical features seen in our study, dyspnea occurred in $64 \%$, altered sensorium in $30 \%$, jaundice in $28 \%$, abdominal pain in $26 \%$, vomiting in $20 \%$, and oliguria in $16 \%$. From North India, an earlier study from this institute and another from Rajasthan showed that the most common presenting symptom was dyspnea in $42 \%$ and $83 \%$, respectively. In contrast, in two other studies from South India, abdominal symptoms (nausea and vomiting and abdominal pain) predominated in $54 \%$ and $58 \%$ of cases, respectively. ${ }^{[14-16,19]}$ The presence of eschar, considered pathognomonic of ST, was in $6 \%$ patients in our study, a figure considerably lower when compared to other Indian studies $(32 \%-46 \%) .{ }^{[14,16,19]}$ Furthermore, the presence of eschar was also low (9.5\%) in a study from the neighboring Himalayan region, Shimla, Himachal Pradesh. ${ }^{[17]}$

Of all the hematological parameters, thrombocytopenia was present in $84 \%$ with severe thrombocytopenia in $38 \%$. The incidence of thrombocytopenia in other studies from the
Asia-Pacific region has varied from $17.3 \%$ to $90 \% .^{[14-23]}$ This fact could be attributable to a difference in the cutoff values for thrombocytopenia as well as variable pathogenicity of the different genotypes causing infection. AKI was seen in $42 \%$ when compared to $11.1 \%-66.7 \%$ from studies from different regions of India. ${ }^{[15-17,19]}$ Data from other Asian countries have shown a much lower incidence of AKI in the range of $3.7 \%-19.2 \%{ }^{[22-24]}$ The reason for this observation could be the different criteria used to define AKI in earlier studies.

ARDS occurred in $66 \%$ in our study and $34 \%$ of our cases required invasive mechanical ventilation. From South India, Varghese et al. reported ARDS in $33.7 \%$ of cases and showed that the requirement for assisted mechanical ventilation predicted mortality independently in the multivariate analysis. ${ }^{[16]}$ Other studies from Asia have reported a much lower incidence of ARDS varying from $8.9 \%$ to $11.1 \%$. ${ }^{[21,24]}$ Of the other complications seen in our study, acute encephalitis was present in $18 \%$ of ST cases as compared to $3.4 \%-13.6 \%$ seen in previous studies from India and other Asian countries, respectively. ${ }^{[14-17,19,24]}$

The median duration of in-hospital stay in this study was 8 days (IQR $=5-11$ days) when compared to an earlier study from our institute, which had a median duration of 6 days (IQR $=4-10$ days). ${ }^{[14]}$ Furthermore, the difference between the mean duration of hospital stay between survivors and nonsurvivors was not statistically significant. On the contrary, in a similar study from Vellore, the mean duration of the hospital stay among survivors was three times longer when compared to nonsurvivors. ${ }^{[5]}$ 
The CFR in the present study was $8 \%$ when compared with two earlier studies from this institute where a reduction in CFR from $16 \%$ to $13.6 \%$ was recorded from 2011 till 2014. ${ }^{[14,25]}$ A similar reduction in the mortality trend over successive years has also been seen in a study from South India. ${ }^{[16]}$ This observation could be explained by an increased awareness of the disease and early institution of doxycycline or azithromycin empirically for the patients presenting with tropical infections. Other Asian countries have reported a low CFR, 6.2\% from Thailand and $0.4 \%$ from Vietnam. ${ }^{[22-24]}$ A possible explanation for this may be the lower incidence of complications in reported cases of ST and presence of strains of lower virulence in these geographical areas.

The mean APACHE II, SOFA, as well as qSOFA scores were higher in the nonsurvivors as compared to survivors with SOFA and qSOFA scores showing statistical significance. On the other hand, Griffith et al. from South India have shown that it is the APACHE II score and not the SOFA score that predicted mortality independently on multivariate analysis. ${ }^{[5]}$ The reason for this paradox may be a small sample size and the fact that in our study only patients admitted to the medical emergency ward and not ICU were included. Thus, studies on cases of ST admitted to emergency wards, ICUs, and general wards with a larger sample size are essential to compare and analyze the superiority of different scoring systems in different areas.

\section{Conclusion}

Among the three CISSs studied, the SOFA score correlated with in-hospital stay duration and mortality, whereas the qSOFA score formed a simple as well as a convenient tool in predicting the mortality in patients of $\mathrm{cST}$ with organ dysfunction(s).

\section{Strength and limitations of the study}

- An observational prospective study

- In the emergency department, SOFA, APACHE II, as well as the qSOFA score, a newer scoring system, were used to assess the outcome

- A study of larger sample size is needed to make a definite conclusion regarding the superiority of one CISS over the other.

\section{Financial support and sponsorship}

Nil.

\section{Conflicts of interest}

There are no conflicts of interest.

\section{RefEREnCes}

1. Watt G. Scrub typhus. In: Warrell DA, Cox TM, Firth JD, editors. Oxford Textbook of Medicine. $5^{\text {th }}$ ed. USA: Oxford University Press; 2010. p. 919-24.

2. Vincent JL, Moreno R, Takala J, Willatts S, De Mendonça A, Bruining H, et al. The SOFA (Sepsis-related organ failure assessment) score to describe organ dysfunction/failure. On behalf of the working group on sepsis-related problems of the European of Intensive Care Medicine. Intensive Care Med 1996;22:707-10.
3. Knaus WA, Draper EA, Wagner DP, Zimmerman JE. APACHE II: Aseverity of disease classification system. Crit Care Med 1985;13:818-29.

4. Seymour CW, Liu VX, Iwashyna TJ, Brunkhorst FM, Rea TD, Scherag A, et al. Assessment of clinical criteria for sepsis: For the third international consensus definitions for sepsis and septic shock (Sepsis-3). JAMA 2016;315:762-74.

5. Griffith M, Peter JV, Karthik G, Ramakrishna K, Prakash JA, Kalki RC, et al. Profile of organ dysfunction and predictors of mortality in severe scrub typhus infection requiring intensive care admission. Indian J Crit Care Med 2014;18:497-502.

6. Furuya Y, Yoshida Y, Katayama T, Yamamoto S, Kawamura A Jr. Serotype-specific amplification of Rickettsia tsutsugamushi DNA by nested polymerase chain reaction. J Clin Microbiol 1993;31:1637-40.

7. Dellinger RP, Levy MM, Rhodes A, Annane D, Gerlach H, Opal SM, et al. Surviving sepsis campaign: International guidelines for management of severe sepsis and septic shock, 2012. Intensive Care Med 2013;39:165-228.

8. ARDS Definition Task Force, Ranieri VM, Rubenfeld GD, Thompson BT, Ferguson ND, Caldwell E, et al. Acute respiratory distress syndrome: The Berlin definition. JAMA 2012;307:2526-33.

9. Khwaja A. KDIGO clinical practice guidelines for acute kidney injury. Nephron Clin Pract 2012;120:c179-84.

10. Sekhon SS, Roy V. Thrombocytopenia in adults: A practical approach to evaluation and management. South Med J 2006;99:491-8.

11. Polson J, Lee WM, American Association for the Study of Liver Disease. AASLD position paper: The management of acute liver failure. Hepatology 2005;41:1179-97.

12. Sharma S, Mishra D, Aneja S, Kumar R, Jain A, Vashishtha VM, et al. Consensus guidelines on evaluation and management of suspected acute viral encephalitis in children in India. Indian Pediatr 2012;49:897-910.

13. Moron CG, Popov VL, Feng HM, Wear D, Walker DH. Identification of the target cells of Orientia tsutsugamushi in human cases of scrub typhus. Mod Pathol 2001;14:752-9.

14. Sharma N, Biswal M, Kumar A, Zaman K, Jain S, Bhalla A, et al. Scrub typhus in a tertiary care hospital in North India. Am J Trop Med Hyg 2016;95:447-51.

15. Sinha P, Gupta S, Dawra R, Rijhawan P. Recent outbreak of scrub typhus in north western part of India. Indian J Med Microbiol 2014;32:247-50.

16. Varghese GM, Trowbridge P, Janardhanan J, Thomas K, Peter JV, Mathews $\mathrm{P}$, et al. Clinical profile and improving mortality trend of scrub typhus in South India. Int J Infect Dis 2014;23:39-43.

17. Mahajan SK, Rolain JM, Kashyap R, Bakshi D, Sharma V, Prasher BS, et al. Scrub typhus in Himalayas. Emerg Infect Dis 2006;12:1590-2.

18. Narvencar KP, Rodrigues S, Nevrekar RP, Dias L, Dias A, Vaz M, et al. Scrub typhus in patients reporting with acute febrile illness at a tertiary health care institution in Goa. Indian J Med Res 2012;136:1020-4.

19. Vivekanandan M, Mani A, Priya YS, Singh AP, Jayakumar S, Purty S, et al. Outbreak of scrub typhus in Pondicherry. J Assoc Physicians India 2010;58:24-8.

20. Park JH, Kim SJ, Youn SK, Park K, Gwack J. Epidemiology of scrub typhus and the eschars patterns in South Korea from 2008 to 2012. Jpn J Infect Dis 2014;67:458-63.

21. Wang YC, Chen PC, Lee KF, Wu YC, Chiu CH. Scrub typhus cases in a teaching hospital in Penghu, Taiwan, 2006-2010. Vector Borne Zoonotic Dis 2013;13:154-9.

22. Hamaguchi S, Cuong NC, Tra DT, Doan YH, Shimizu K, Tuan NQ, et al. Clinical and epidemiological characteristics of scrub typhus and murine typhus among hospitalized patients with acute undifferentiated fever in Northern Vietnam. Am J Trop Med Hyg 2015;92:972-8.

23. Zhang M, Zhao ZT, Wang XJ, Li Z, Ding L, Ding SJ, et al. Scrub typhus: Surveillance, clinical profile and diagnostic issues in Shandong, China. Am J Trop Med Hyg 2012;87:1099-104.

24. Thipmontree W, Tantibhedhyangkul W, Silpasakorn S, Wongsawat E, Waywa D, Suputtamongkol Y, et al. Scrub typhus in Northeastern Thailand: Eschar distribution, abnormal electrocardiographic findings, and predictors of fatal outcome. Am J Trop Med Hyg 2016;95:769-73.

25. Kumar V, Kumar V, Yadav AK, Iyengar S, Bhalla A, Sharma N, et al. Scrub typhus is an under-recognized cause of acute febrile illness with acute kidney injury in India. PLoS Negl Trop Dis 2014;8:e2605. 